\title{
Cenários Prospectivos para a Inovação Social
}

\author{
Prospective Scenarios for Social Innovation \\ Escenarios Prospectivos para la Innovación Social
}

Recebido: 15/04/2021 | Revisado: 21/04/2021 | Aceito: 25/04/2021 | Publicado: 09/05/2021

\author{
Alequexandre Galvez de Andrade \\ ORCID: https://orcid.org/0000-0002-5847-7616 \\ Instituto Federal de Educação, Ciência e Tecnologia de São Paulo, Brasil \\ E-mail: aleq.galvez@ifsp.edu.br \\ Fernando Rodrigues de Amorim \\ ORCID: https://orcid.org/0000-0003-1618-6316 \\ Universidade Estadual Paulista, Brasil \\ E-mail:fernando.amorim@unesp.br \\ Nélio Fernando dos Reis \\ ORCID: https://orcid.org/0000-0002-1694-4917 \\ Instituto Federal de Educação, Ciência e Tecnologia de São Paulo, Brasil \\ E-mail:nelio.reis@ifsp.edu.br
}

\begin{abstract}
Resumo
A Inovação Social tem seu campo fundamentado na criação de soluções frente às necessidades sociais, impulsionada por diversos agentes que se organizam sob os mais diferentes tipos jurídicos, ampliando a ação da inovação social (IS) de um campo local para nacional ou internacional. Estas transformações sociais impõem desafios aos gestores que passam a considerar as IS como uma nova dimensão para a estratégia e o planejamento nas organizações, exigindo a elaboração de cenários futuros. $\mathrm{O}$ objetivo deste estudo foi desenvolver cenários prospectivos para a inovação social no curto prazo. A abordagem é qualitativa e quantitativa, utilizando-se da Lógica Paraconsistente Anotada Evidencial ${ }^{1}$ (lógica E $\tau$ ). Foi elaborado um questionário, contendo 58 questões, sendo 57 fechadas e 1 aberta. Para validar o questionário a priori foram consultados 3 especialistas, as proposições foram confirmadas por meio de rodadas Delphi e teste de consistência interna medida pelo Alpha de Cronbach $^{2}$. A amostra foi constituída por 26 participantes, distribuídos em dois grupos, grupo A formado por 13 especialistas e grupo B por 13 profissionais gestores ou que tenham participado de projetos sociais. Para medir se existe diferença estatística significativa entre os grupos, foi utilizado o teste de Levene $^{3}$. Os resultados indicam que não existe diferença significativa entre os grupos e validam o modelo para o cenário de curto prazo, que contém os fatores distribuídos em transformação, inovação, geografia da inovação, atores e processos.
\end{abstract}

Palavras-chave: Cooperativismo; Cenários prospectivos; Inovação; Inovação social; Lógica E $\tau$.

\begin{abstract}
Social Innovation has its field based on the creation of solutions in face of social needs, driven by several agents that organize themselves under the most different legal types, expanding the action of Social Innovation (SI) from a local to national or international field. These social changes impose challenges for managers who start to consider SI as a new dimension for strategy and planning in organizations, requiring the elaboration of future scenarios. The aim of this study was to develop prospective scenarios for social innovation in the short-term. The approach is qualitative and quantitative, using Evidential Annotated Paraconsistent Logic (E $\tau$ logic). A questionnaire was prepared, containing 58 questions, 57 of which were closed and 1 was open. In order to validate the questionnaire a priori, 3 specialists were consulted, the proposals were confirmed through Delphi rounds and an internal consistency test measured by Cronbach's Alpha. The sample consisted of 26 participants, distributed in two groups, group A formed by 13 specialists and group B by 13 professional managers or who have participated in social projects. To measure whether there is a statistically significant difference between the groups, the Levene test was used. The results indicate that there is no significant difference between the groups and validate the short-term models, which contain the factors distributed in transformation, innovation, geography of innovation, actors and processes.
\end{abstract}

Keywords: Cooperativism; Prospective scenarios; Innovation; Social innovation; Logic E $\tau$.

\footnotetext{
${ }^{1}$ Lógica Paraconsistente Anota Evidencial: as teorias paraconsistentes permitem teoremas de contradição, em uma mesma proposição pode assumir graus de crença ou descrença. São consideradas não triviais e adequadas ao conhecimento complexo (Costa \& Abe, 2000).

${ }^{2}$ Alpha de Cronbach: utilizado para quantificar a confiabilidade de um questionário, trata-se de um índice de homogeneidade da amostra (Brown, 2002).

${ }^{3}$ Teste de Levene: demonstra a homogeneidade das variações, sendo considerado um instrumento robusto e poderoso (Gastwirth, Gel, \& Miao, 2009).
} 


\begin{abstract}
Resumen
La Innovación Social tiene su campo basado en la creación de soluciones frente a las necesidades sociales, impulsadas por varios agentes que se organizan bajo los tipos legales más diferentes, expandiendo la acción de Innovación Social (IS) de un campo local a un campo nacional o internacional. Estos cambios sociales imponen desafíos para los gerentes que comienzan a considerar el SI como una nueva dimensión para la estrategia y la planificación en las organizaciones, lo que requiere la elaboración de escenarios futuros. El objetivo de este estudio fue desarrollar escenarios prospectivos para la innovación social a corto plazo. El enfoque es cualitativo y cuantitativo, utilizando lógica paraconsistente anotada evidencial (lógica $\mathrm{E} \tau$ ). Se preparó un cuestionario que contenía 58 preguntas, 57 de las cuales estaban cerradas y 1 estaba abierta. Para validar el cuestionario a priori, se consultó a 3 especialistas, las propuestas se confirmaron mediante rondas Delphi y una prueba de consistencia interna medida por Alpha de Cronbach. La muestra consistió en 26 participantes, distribuidos en dos grupos, el grupo A formado por 13 especialistas y el grupo B por 13 gerentes profesionales o que han participado en proyectos sociales. Para medir si existe una diferencia estadísticamente significativa entre los grupos, se utilizó la prueba de nivel. Los resultados indican que no existe una diferencia significativa entre los grupos y validan los modelos a corto plazo, que contienen los factores distribuidos en transformación, innovación, geografía de innovación, actores y procesos.
\end{abstract}

Palabras clave: Cooperativismo; Escenarios prospectivos; Innovación; Innovación social; Lógica E $\tau$.

\title{
1. Introdução
}

A Inovação Social (IS) surge em um contexto para atender às necessidades sociais, inerentes a qualquer sistema econômico, por isso atua de maneira articulada com diversos agentes, setor público, setor privado, sociedade civil e outros.

Independentemente do nível de desenvolvimento econômico, as inovações sociais estarão presentes e podem vir revestidas por diversos tipos jurídicos, como cooperativas, organizações sociais, empresas privadas e outros. Nos países desenvolvidos em grande parte as inovações sociais foram produzidas pela iniciativa privada e devem ser consideradas como uma nova dimensão pelos gestores (Drucker, 1987).

O reconhecimento da importância destas instituições estimulou o surgimento de uma economia social, que vem crescendo ao longo dos anos por meio do terceiro setor. Por isso, a capacidade de se antecipar a problemas futuros torna-se um fator de sobrevivência para as sociedades, e em sentido estrito para as ações sociais afirmativas, que buscam em sua essência reduzir as desigualdades.

As desigualdades devem ser compreendidas de forma ampla, como acesso à educação, saúde, moradia, liberdade, cultura e outros. A garantia do acesso e qualidade a estes produtos e serviços, reduziriam problemas sociais como violência, consumo de álcool, homicídios e outros (Wilkinson \& Pickett, 2009).

Diante dessas diversidades e necessidades que serão observadas de forma mais direta em países subdesenvolvidos como o Brasil, a inovação social compreende a demanda mais específica de determinados grupos, para fomentar o empoderamento destas pessoas, por este motivo, uma inovação social jamais deve estar escorada na estigmatização de grupos sociais.

Esta dimensão a ser observada por gestores de diversos tipos de organizações sublinham a necessidade de prever cenários futuros, que é um desafio para gestores, que precisam agir no presente para construir o futuro das organizações. A falta de responsabilidade social pode ser considerada uma fraqueza competitiva como a observada no setor sucroenergético brasileiro (Mannarelli Filho, Donadon, Pigatto, Queiróz, \& Baptista, 2021).

A capacidade em prever cenários para IS é ainda mais complexa devido à frequência das convulsões sociais. Esta complexidade deve-se em grande parte às inovações e a internacionalização das economias, que por meio da computação nas nuvens e a velocidade com que as informações circulam e as pessoas se relacionam, estão promovendo a mudança de foco nas organizações que passa a ser centrada no consumidor (Prahalad \& Ramaswamy, 2004).

Neste contexto, os consumidores estão mais empoderados, com maior capacidade de negociação, isto estimula o surgimento de novos produtos e serviços ou melhorias em produtos e serviços existentes com foco no consumidor (Prahalad \& Ramaswamy, 2004). 
Diante do exposto, os gestores formulam proposições para criarem cenários prospectivos com o propósito de orientar as organizações, a fim de apoiar a estratégia e o planejamento. A negação deste olhar para o futuro, leva organizações e pessoas ao fracasso (Schwartz, 1996). Neste sentido, pergunta-se: Quais são as proposições da inovação social que devem ser formuladas a fim de construir cenários futuros para o curto prazo?

A inovação social por ser multissetorial e interdisciplinar forma um sistema complexo de interações, para desenvolver estes cenários foi utilizada a lógica paraconsistente anotada $(\mathrm{E} \tau)$. Trata-se de um grupo de lógica não clássica que aceita graus de certeza e contradição na mesma proposição, o que é inerente ao conhecimento humano.

O objetivo deste estudo foi desenvolver cenários prospectivos de curto prazo para a inovação social. Para atingir o objetivo foi aplicado um questionário contendo 58 perguntas, sendo 57 fechadas e uma aberta. O questionário foi avaliado a priori por três especialistas e analisada a consistência interna das respostas por meio do Alpha de Cronbach. Os participantes foram separados em dois grupos, grupo A especialistas acadêmicos e grupo B especialistas gestores ou profissionais que tenham participado de projetos sociais. Foram enviadas 42 mensagens e recebidas 26 respostas, índice considerado positivo para rodadas Delphi. Após analisados os apontamentos na questão aberta, não foi necessário efetuar novas rodadas Delphi.

Para analisar os dados foi aplicado a Lógica E $\tau$, com os conectores or e and, calculado o grau de certeza e contradição normalizados para validar a adequação dos modelos, e avaliado se existe diferença estatística significativa entre os grupos A e $\mathrm{B}$, para isto, utilizou o teste de Levene. Os resultados indicam que não há diferenças estatísticas relevantes entre os grupos e validam o cenário de curto prazo, confirmando os fatores transformação, inovação, geografia da inovação, atores e processos.

\section{Metodologia}

Este estudo possui abordagem qualitativa e quantitativa. A abordagem quantitativa utiliza-se de um conjunto de dados numéricos para confirmar hipóteses e teorias por meio de processos estruturados e não permite que nenhuma etapa seja olvidada (Sampieri, Collado, \& Lucio, 2010). Quanto a pesquisa qualitativa, o autor supracitado elenca que se utiliza um conjunto de informações sem medição numérica a fim de formar conceitos e perguntas para o processo de investigação. Portanto, na pesquisa qualitativa têm-se proposições a serem confirmadas. A natureza quantitativa deste estudo se restringe à identificação da homogeneidade entre grupos de participantes, sendo formados pelos grupos A e B, conforme apresentado no item Amostra. Este projeto foi aprovado pelo Conselho Nacional de Ética em Pesquisa (CONEP), Plataforma Brasil, por meio do parecer 4.423 .746 de 26 de novembro de 2020.

A lógica E $\tau$, por ter abordagem qualitativa tem cunho descritivo (Sanches, Marietto, \& Paixão, 2011). Entretanto, dispõe de processos para validar os fatores e proposições, a fim de obter premissas para a composição de cenários futuros (Reis, 2014).

Os cenários foram construídos de maneira independente, baseado na opinião dos especialistas, sem intervenção do pesquisador, uma característica da pesquisa descritiva que "busca especificar as propriedades, as características e o perfil de pessoas, grupos, comunidades, processos, objetos ou qualquer fenômeno que se submeta a análise" (Sampieri et al., 2010, p.80).

Para validar os cenários foi utilizado a Lógica E $\tau$ que é considerado um tipo de lógica não clássica com seus próprios procedimentos, compondo um método diversificado e aplicável a diversos contextos, obtendo o grau de certeza e contradição, o que está mais aderente a casos reais de tomada de decisão (Carvalho, Brustein, \& Abe, 2011).

Esta pesquisa também é do tipo correlacional. Este tipo de pesquisa correlaciona variável para responder determinadas perguntas (Sampieri et al., 2010) estas variáveis correspondem ao grupo A e B. O grupo A é formado por especialistas e o 
grupo B por gestores ou conhecedores de projetos sociais. O objetivo desta separação é compreender se há diferença estatística significativa entre o que está sendo pesquisado e praticado em termos de inovação social.

Neste estudo a interdisciplinaridade é importante, pois em um processo decisório deve-se levar em consideração o conhecimento complexo. Desta forma não é possível separar a parte do todo, "a complexidade é a união entre a unidade e a multiplicidade" e cada vez mais possui este caráter multidimensional, ou seja, da união de várias habilidades (Morin, 2000, p. $38)$.

\subsection{Amostra}

Não há consenso quanto ao aspecto amostral para aplicação da técnica Delphi. Para Astigarraga (2003), a proposta está entre 7 e 30 participantes, já para Velez Pareja (2003), deve estar entre 4 ou 5 participantes. Para Hamilton e Breslawski (1996) a amostra pretendida deve ser multiplicada por 5 e como exemplo em uma pesquisa com envio para 150 especialistas, apenas 32 concluíram o processo e identificou a redução na participação à medida que foram ocorrendo as rodadas. $\mathrm{O}$ espaço amostral deve estar centrado na qualidade e não na quantidade dos especialistas. Para este trabalho, foi definida a amostra mínima de 20 participantes, que é a média dos pontos máximos entre os dois teóricos e condiz com o mapeamento realizado para definir os especialistas.

A qualidade dos especialistas pode ser identificada por trabalhos acadêmicos publicados, titulação, experiência profissional, conhecimento profundo do assunto (Reis, 2014; Sanches, Marietto, \& Paixão, 2011).

Os especialistas foram identificados por meio de grupos de pesquisa autorizados pela CAPES e do cadastro no LinkedIn, foram observados a qualificação, atividade e currículo do participante.

$\mathrm{Na}$ Lógica $\mathrm{E} \tau$, por se tratar de sistemas especialistas, é dada a importância para a qualidade e não quantidade do espaço amostral (Abe \& Costa, 1992; Carvalho \& Abe, 2011; Reis, Oliveira, Abe, \& Andrade, 2019). Neste sentido a Microrregião de Jundiaí dada a sua importância em termos de especialização e tecnologia, obtém uma heterogeneidade importante para a qualidade das especializações. Desta forma os participantes da pesquisa deveriam conhecer a região de Jundiaí ou regiões que possuem características equivalentes.

\subsection{Questionário}

O questionário foi elaborado com base no estudo de Tardif e Harrisson (2005), vinculados ao Crises (Centre de Recherche Sur Les Innovations Sociales), uma organização interuniversitária que estuda as transformações e inovações sociais, sediada em Quebec, Canadá.

Exceto pelas perguntas de identificação, demográfica e questão aberta, as demais perguntas foram realizadas com base na escala Likert que representa uma escala de atitude e juízos (Likert, 1932), dispondo escala intervalar e ordinal (Budiaji, 2013). Para este questionário foram utilizadas as escalas com as devidas pontuações para efetuar as análises estatísticas e aplicação da lógica E $\tau$, seguindo o enfoque afirmativo proposto por Sampieri et. al. (2010). Sendo, concordo totalmente - 5 pontos; concordo parcialmente - 4 pontos; neutro - 3 pontos; discordo parcialmente - 2 pontos e discordo totalmente - 1 ponto.

A questão aberta número 58, faz com que o questionário seja dinâmico, pois serão conduzidas rodadas Delphi para buscar identificar temas relevantes para cenários prospectivos. Este método promove o debate entre os especialistas que emitem pareceres e juízos sobre o tema, também pode ser utilizado em cenários que demandam interdisciplinaridade (Rozados, 2015). Esta discussão foi conduzida por meio da publicação dos parâmetros, sem que haja relação entre os participantes. Isto permite a isenção da pesquisa e identificação entre os pares. As rodadas foram conduzidas observado o exposto por Hamilton e Breslawski (1996). 
A avaliação do questionário foi realizada por especialistas no assunto. O Especialista 1 é historiador com foco em história estadunidense e relações sociais. O Especialista 2, tem formação em administração, com trabalhos publicados em periódicos sobre mobilidade urbana e temas relacionados às relações sociais. $\mathrm{O}$ especialista 3 , tem formação em administração pública, com foco em políticas públicas e experiência em projetos sociais. Foram criados três conceitos para avaliação do questionário, sendo: atribuídos três conceitos: $\mathrm{Y}=$ Concordo, $\mathrm{YI}=$ concordo parcialmente e Não = Não concordo, caso seja atribuído dois YI ou Não ou a combinação entre eles, a questão seria eliminada. A consulta de especialistas pode ser considerada como matéria prima para questionários (Sampieri et al., 2010). Após concluída as rodadas, nenhuma questão foi eliminada apenas aperfeiçoadas quanto à redação.

Para validar a consistência interna do questionário, a fim de estimar a confiabilidade, foi calculado o Alpha de Cronbach por meio do software SPSS $\circledR_{25}^{25}$, que trata de um índice de homogeneidade da amostra (James Dean Brown, 2002).

Para pesquisas exploratórias o índice de 0,6 pode ser utilizado, em escalas múltiplas deve exceder a 0,70 (Hair, Black, Babin, Anderson, \& Tatham, 2009), valores acima de 0,81 possuem uma consistência quase perfeita (Landis \& Koch, 1977).

\subsection{Coleta de Dados}

O questionário foi elaborado usando a ferramenta Google forms, como parâmetros foi definido a obrigatoriedade do email para que fosse possível realizar as rodadas, as perguntas demográficas e, por fim, as perguntas do questionário baseada nos fatores, todas configuradas como obrigatórias para todas as linhas. Não foi necessário refazer as rodadas, pois não ocorreram apontamentos adicionais.

O questionário foi encaminhado para 42 pessoas, por meio de e-mail e mensagem no LinkedIn para o público selecionado, ambos atendem os requisitos demonstrados no escopo da amostragem. Foram obtidas 26 respostas, um índice de 50\% de retorno, o que é positivo frente ao índice observado em outros estudos (Hamilton \& Breslawski, 1996). Destas 26 respostas, foram analisadas e classificadas em dois grupos, sendo 13 especialistas acadêmicos e 13 gestores ou profissionais que atuam em projetos sociais, respeitando o recorte amostral.

\subsection{Aplicação da Lógica $E \tau$}

O operador MÁX do reticulado [0,1] x [0,1], associado à Lógica E $\tau$, é para ser aplicado a um grupo de $\mathrm{n}$ anotações $(\mathrm{n} \geq 1)$. Ele atua de modo a maximizar o grau de certeza $\left(\mathrm{G}_{\text {cert }}=\mu-\lambda\right)$ desse grupo de anotações, selecionando a melhor evidência favorável (maior valor de $\mu$ ) e a pior evidência contrária (menor valor de $\lambda$ ), não sendo aplicado em situações em que os dois ou mais itens considerados não são todos determinantes, bastando que um deles tenha condição favorável para se considerar satisfatório (Carvalho; Abe, 2011). Ele é definido da seguinte forma: MÁX $\left.\left\{\mu_{1} ; \lambda_{1}\right),\left(\mu_{2} ; \lambda_{2}\right), \ldots\left(\mu_{n} ; \lambda_{n}\right)\right\}=$ (máx $\left\{\mu_{1}, \mu_{2}, \ldots, \mu_{n}\right\} ;$ mín $\left\{\lambda_{1}, \lambda_{2}, \ldots \lambda_{n}\right\}$ ) (Abe \& Costa, 1992; Carvalho \& Abe, 2011; Reis, Oliveira, Abe, \& Andrade, 2019). No caso do operador MÍN, utiliza-se a pior evidência Favorável (Carvalho; Abe, 2011).

No resultado, após aplicação das regras de maximização e de minimização, adota-se como limites de verdade e de falsidade como nível de exigência. Desta forma, tem-se evidência favorável ou contrária a viabilidade do estudo em tela, se houver um grau de certeza em módulo igual ou maior que 0,6. De forma resumida: $\mathrm{G}_{\text {cert }} \geq 0,6 \rightarrow$ Verdade (V), ou seja, a aquisição do ativo é VIÁVEL; $\mathrm{G}_{\text {cert }} \leq-0,6 \rightarrow$ Falsidade (F), ou seja, a aquisição do ativo é INVIÁVEL; $-0,6<\mathrm{G}_{\text {cert }}<0,6 \rightarrow$ Confuso (T), ou seja, DÚVIDA, devendo haver aprofundamento das informações (Reis, 2014).

Para montar o Reticulado representado por um Quadrado Unitário no Plano Cartesiano (QUPC) de cinco fatores, foi utilizado o conectivo OR e AND. OR é usado para maximizar o maior valor das entradas e AND para minimizar o menor valor das entradas. A figura 1, demonstra a lógica para cinco fatores. 
Figura 1 - Redes Lógicas para Cinco Fatores.

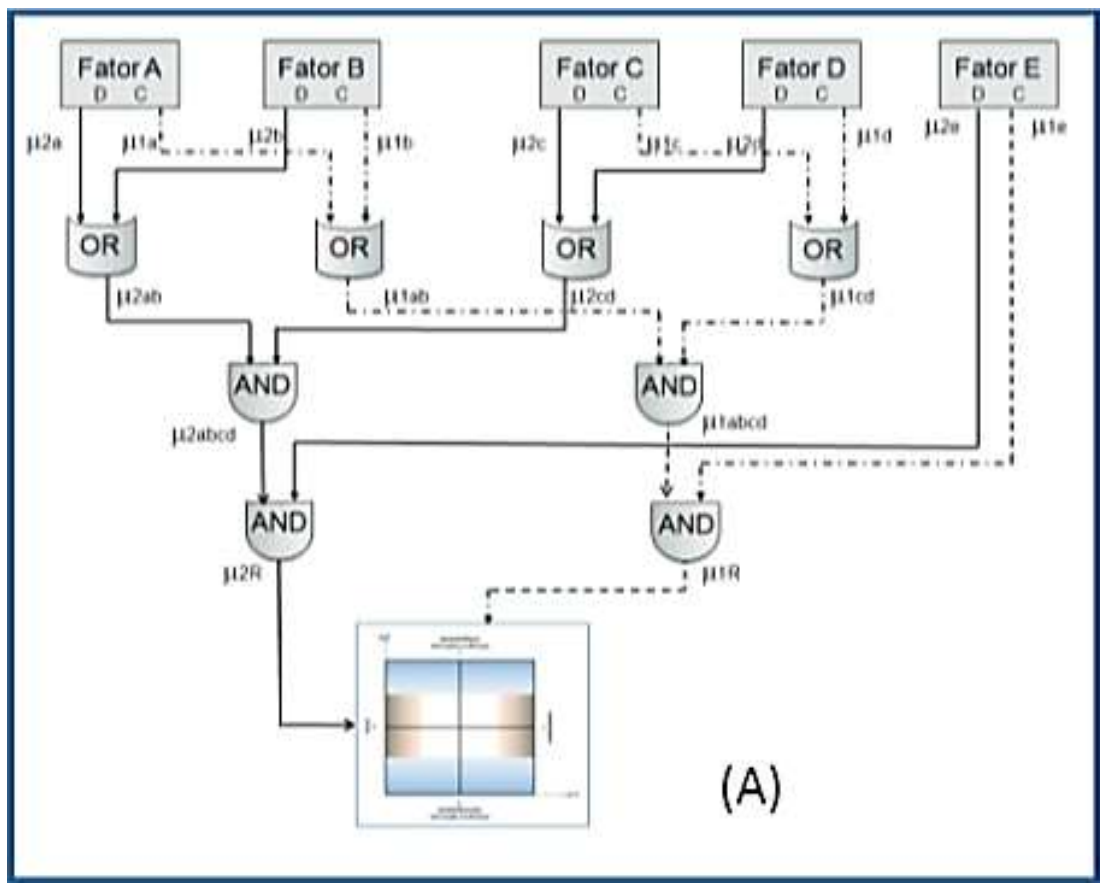

Fonte: Sanches, Meireles e Sordi (2011, p. 9).

$\mathrm{Na}$ avaliação de questionários por meio da lógica $\mathrm{E} \tau$, deve-se equilibrar o elemento neutro tanto para os grupos que concordam como os que discordam, usando a proposta de Macnaughton (1996), esse equilíbrio é realizado partilhando 50\% do elemento neutro para o grupo Discordo e Concordo, de tal forma que a soma de Discordo totalmente (DT) Discordo Parcialmente (D) + Neutro + Concordo Parcialmente (C) + Concordo Totalmente (CT), depois de realizado o equilíbrio, seja igual a DT + D + C + CT. Calcula-se de acordo com a fórmula (1).

$$
D_{p}=D T+D+I / 2 \quad C_{p}=C+C T+I / 2
$$

Quanto à normalização (n) do grau de contradição e certeza, utilizam-se respectivamente as equações Gctn (2) e Gcn (3) (Sanches, Meireles, \& Sordi., 2011, p. 11).

$$
\begin{aligned}
& G \operatorname{ctn}=\frac{G c t-(-1)}{1-(-1)}=\frac{G c t+1}{2}=\frac{(\mu 1-\mu 2)+1}{2} \\
& G c n=\frac{G c-(-1)}{1-(-1)}=\frac{G c+1}{2}=\frac{(\mu 1+\mu 2)}{2}
\end{aligned}
$$

\subsection{Teste de Levene}

Para identificar a diferença entre grupos foi realizado o teste de Levene, que demonstra a homogeneidade das variações, sendo considerado um instrumento robusto e poderoso (Gastwirth, Gel, \& Miao, 2009), neste teste assume duas hipóteses, nula e alternativa, desdobrada em $\mathrm{H0}=$ não há diferenças de variância entre os grupos e H1 = há diferença de variância entre os grupos (Levene, 1960). Assumindo um índice de confiança de 95\%, se $\mathrm{H} \leq 0,05$ assume H1, há diferenças 
entre os grupos de especialistas acadêmicos (A) e especialistas gestores (B). Se $\mathrm{H} \geq 0,05$, não há diferenças entre os grupos, assumindo H0, que indica homogeneidade entre os grupos A e B. Para calcular foi utilizado o software SPSS ® 25.

\section{Resultados e Discussão}

A Tabela 1, apresenta a distribuição dos especialistas por gênero, sendo 8 mulheres com 30,7\% e 18 homens com $69,3 \%$ do total.

Tabela 1 - Frequência de Gênero.

\begin{tabular}{l|c|c|c}
\hline \multicolumn{1}{c|}{ Gênero } & Frequência & Porcentagem & $\begin{array}{c}\text { Porcentagem } \\
\text { acumulada }\end{array}$ \\
\hline Feminino & 8 & 30,7 & 30,7 \\
Masculino & 18 & 69,3 & 100,0 \\
\hline Total & 26 & 100,0 & \\
\hline
\end{tabular}

Fonte: Autores (2020).

Em relação à faixa etária as frequências são heterogêneas, a maior faixa está entre 30 a 39 anos, com 30,8\% do total, seguida da faixa de 40 a 49 anos, com $23,1 \%$ do total, maior que 59 anos totalizaram 19,2\%, e as faixas de 20 a 29 anos e de 50 a 59 anos, representaram respectivamente $15,4 \%$ e $11,5 \%$. A Tabela 2 indica as faixas etárias.

Tabela 2 - Faixa etária dos participantes.

\begin{tabular}{l|c|c|c}
\hline \multicolumn{1}{c|}{ Faixa etária } & Frequência & Porcentagem & $\begin{array}{c}\text { Porcentagem } \\
\text { acumulada }\end{array}$ \\
\hline 20 a 29 anos & 4 & 15,4 & 15,4 \\
30 a 39 anos & 8 & 30,8 & 46,2 \\
40 a 49 anos & 6 & 23,1 & 69,3 \\
50 a 59 anos & 3 & 11,5 & 80,8 \\
maior que 59 anos & 5 & 19,2 & 100,0 \\
\hline Total & 26 & 100,0 & \\
\hline
\end{tabular}

Fonte: Autores (2020).

A maior parte dos participantes possui título de mestrado e doutorado, com 46,1\% do total, os que possuem especialização representam $15,5 \%$, graduação $26,9 \%$ e os demais somam $11,5 \%$ do total. O que é recomendável para a qualidade da amostra, pois a pesquisa foi distribuída em dois grupos, de um lado temos os acadêmicos do tema e do outro lado os gestores que atuam em projetos com finalidade de inovação social. A Tabela 3 indica o grau de escolaridade. 
Tabela 3 - Grau de escolaridade.

\begin{tabular}{c|c|c|c}
\hline Grau de Escolaridade & Frequência & Porcentagem & $\begin{array}{c}\text { Porcentagem } \\
\text { acumulada }\end{array}$ \\
\hline Doutorado & 5 & 19,2 & 19,2 \\
Ensino Médio Completo & 1 & 3,8 & 23,0 \\
Ensino Superior Completo & 7 & 26,9 & 49,9 \\
Ensino Superior incompleto & 2 & 7,7 & 57,6 \\
Especialização & 4 & 15,5 & 73,1 \\
Mestrado & 7 & 26,9 & 100,0 \\
\hline Total & 26 & 100,0 & \\
\hline
\end{tabular}

Fonte: Autores (2020).

As áreas de formação são heterogêneas, o que condiz com o tema inovação social, que é multidisciplinar pelas suas características baseadas nas necessidades com foco no social e, por consequência, estas necessidades estão relacionadas a sistemas complexos. As áreas de ciências sociais aplicadas e ciências humanas representaram 54,1\% do total, com 14 participantes. A Tabela 4 apresenta a distribuição dos participantes por área de formação acadêmica.

Tabela 4 - Áreas de formação.

\begin{tabular}{l|c|c|c}
\hline \multicolumn{1}{c|}{ Área } & Frequência & Porcentagem & $\begin{array}{c}\text { Porcentagem } \\
\text { acumulada }\end{array}$ \\
\hline Ciências Agrárias & 1 & 3,8 & 3,8 \\
Ciências Biológicas & 1 & 3,8 & 7,6 \\
Ciências Exatas e da Terra & 3 & 11,5 & 19,1 \\
Ciências Humanas & 6 & 23,2 & 42,3 \\
Ciências Sociais Aplicada & 8 & 30,9 & 73,2 \\
Engenharias & 1 & 3,8 & 77,0 \\
Linguística, Letras e Arte & 3 & 11,5 & 88,5 \\
Outros & 3 & 11,5 & 100,0 \\
\hline Total & 26 & 100,0 & \\
\hline
\end{tabular}

Fonte: Autores (2020).

A construção de um questionário baseado em escalas permitem várias possibilidades de graus de respostas, ao sumarizar esses valores é preciso medir a consistência interna, a fim de validar os constructos (George \& Mallery, 2018), que no caso em tela denominam-se fatores, proposições e premissas. Esta abordagem permite validar a construção, tendo como base a correlação entres os itens (James Dean Brown, 2002).

Para pesquisas exploratórias o índice de 0,6 pode ser utilizado, em escalas múltiplas deve exceder a 0,70 (Hair et al., 2009), valores acima de 0,81 possuem uma consistência quase perfeita (Landis \& Koch, 1977), o índice do questionário deste trabalho foi de 0,892 , demonstrando uma consistência quase perfeita, conforme a Tabela 5 . 
Tabela 5 - Alfa de Cronbach.

\begin{tabular}{c|c|c}
\hline Alfa de Cronbach & $\begin{array}{c}\text { Alfa de Cronbach com base em itens } \\
\text { padronizados }\end{array}$ & N de itens \\
\hline 0,862 & 0,892 & 52 \\
\hline
\end{tabular}

Fonte: Autores (2020).

\subsection{Cenários Prospectivos de Curto Prazo}

O Curto prazo (CP) para este estudo compreende o período de até dois anos. O Grau de certeza $\mathrm{G}_{\text {cert }}$, foi calculado pela diferença ente $\mu 1$ e $\mu 2$, Tabela 6 , nota-se que as premissas crises; ruptura para novas formas de trabalho, estimuladas pela tecnologia e descontinuidade de modelos regionais, com índices de 0,35, 0,58 e 0,15, respectivamente, foram consideradas como dúvida, conforme tabela 6. Estas premissas estão associadas ao fator transformação na proposição do contexto. Ainda no fator transformação, na proposição econômica, foram excluídas as premissas adaptação por questões tecnológicas, ambientais e outras $(0,42)$ e mudanças nas relações sociais $(0,50)$. Nas proposições aspectos sociais, foram excluídas as premissas Exclusão social $(0,54)$ e ações por tentativa $(0,50)$.

O fator transformações diz respeito às proposições relacionadas ao ambiente em que decorrem as necessidades e oportunidades para a inovação social (Tardif \& Harrisson, 2005), os especialistas consideraram que no curto prazo deve-se ter como premissa os fatores do índice de desenvolvimento humano (IDH), as mudanças nas relações de trabalho e a marginalização provocada pela separação em grupos sociais.

O IDH trata de uma medida mais ampla que o PIB Per capita, possui três dimensões Saúde, Educação e renda, estas representam as medidas básicas que foram ajustadas em 2010, para obter um retrato mais fiel da desigualdade, ampliando para os indicadores de desigualdade de gênero (IDG), índice de pobreza multidimensional (IPM) (PNUD Brasil, 2020). Esta elasticidade do IDH permite identificar que o crescimento isolado não é capaz de avaliar o desenvolvimento humano. Economias com o mesmo PIB per capita possuem IDH diferentes, no campo das políticas nacionais pode ser utilizado para questionar se estas estão produzindo desenvolvimento (Lind, 2014). Ao pontuarem como uma medida viável os especialistas realçam pelo grau de certeza de que estas dimensões para o curto prazo são mais importantes que as crises, relações sociais, mudanças tecnológicas.

Isto demonstra que no curto prazo, o foco é na operacionalidade com ganhos de eficiência e eficácia (Papakostas, Papachatzakis, Xanthakis, Mourtzis, \& Chryssolouris, 2010) nos moldes das bases do IDH, nas mudanças das relações de trabalho e na separação de estratos da sociedade em grupos superiores e inferiores.

No fator Inovação, foi refutada a ação por tentativa, estas ações permitem a abstração de que há falhas no planejamento estratégico e, consequentemente, podem representar gastos desnecessários que conduziriam a perda de eficiência. Em relação ao fator geografia da inovação foi refutada a escala nacional e internacional, como o CP está direcionado a operacionalidade, o foco na escala local tem mais aceitação pelos especialistas.

As demais premissas viáveis podem ser sintetizadas no conhecimento, apoio de políticas públicas, legislação que ampare estas atividades, parceria público privada, tecnologias sociais, interesse coletivo, bem comum, formação de comitês, oportunidades geradas pelas redes sociais e como restrições a serem mitigadas estão as incertezas políticas, sociais e tecnológicas, restrições externas e regulação institucional.

Após as exclusões das premissas do $\mathrm{G}_{\text {cert }}=$ Dúvida e normalização, o grau de certeza foi de 0,95 (0,93 antes do ajuste) e o grau de contradição foi de 0,48 (0,47 antes do ajuste), apresentando uma aderência substancial e no grau de contradição demonstrou consistência, confirmando a qualidade das premissas para os cenários prospectivos de curto prazo, conforme escala proposta por Davis (1976). 
Depreende-se que todos os fatores Transformação, Inovação, Geografia da Inovação, atores e processos foram validados, indicando a adequação dos cenários para o planejamento CP.

O teste de Levene apresentou homogeneidade entre os grupos, exceto pela questão P29 (t $(24)=2,265 ; \mathrm{p}<0,05)$, referente ao fator geografia da inovação, proposição organização e premissa institucional, em que se confirmou a hipótese H1, demonstrando que há diferenças entre os Grupos A e B, respectivamente com médias de 3,69 e 4,46, indicando um estado mais neutro por parte dos acadêmicos quanto à institucionalização da inovação social. Esta informalidade tem dois recortes. O primeiro diz respeito à informalidade com o objetivo muitas vezes de evitar custos iniciais com o projeto, que se torna formal à medida que ganha escala e esteja sendo aplicado (Choi \& Thum, 2005). O segundo está relacionado às estratégias emergentes, que embora não tenham sido planejadas, são importantes para atingir os objetivos pretendidos (Mintzberg, 1987).

Tabela 6 - Aplicação Lógica Et - Curto Prazo.

\begin{tabular}{|c|c|c|c|c|c|c|c|c|c|c|c|c|c|c|}
\hline \multirow{2}{*}{ Fator } & \multirow{2}{*}{ Proposição } & \multicolumn{6}{|c|}{ Frequência } & \multicolumn{3}{|c|}{ Entre (r) } & \multicolumn{4}{|c|}{ Índice ( $\left.G_{\text {cert }}\right)$} \\
\hline & & 1 & 2 & 3 & 4 & 5 & $\Sigma$ & $\mathrm{Dp}$ & $\mathrm{Cp}$ & $\Sigma$ & $\mu_{1}$ & $\mu_{2}$ & $\mathrm{G}_{\text {cert }}$ & Decisão \\
\hline Transformação & P1 & 5 & 2 & 3 & 3 & 13 & 26 & 8,5 & 17,5 & 26 & 0,67 & 0,33 & 0,35 & Dúvida \\
\hline Transformação & P2 & 1 & 4 & 1 & 11 & 9 & 26 & 5,5 & 20,5 & 26 & 0,79 & 0,21 & 0,58 & Dúvida \\
\hline Transformação & P3 & 4 & 5 & 4 & 9 & 4 & 26 & 11,0 & 15,0 & 26 & 0,58 & 0,42 & 0,15 & Dúvida \\
\hline Transformação & P4 & 2 & 1 & 1 & 8 & 14 & 26 & 3,5 & 22,5 & 26 & 0,87 & 0,13 & 0,73 & Viável \\
\hline Transformação & P5 & 2 & 4 & 3 & 2 & 15 & 26 & 7,5 & 18,5 & 26 & 0,71 & 0,29 & 0,42 & Dúvida \\
\hline Transformação & P6 & 2 & 2 & 5 & 6 & 11 & 26 & 6,5 & 19,5 & 26 & 0,75 & 0,25 & 0,50 & Dúvida \\
\hline Transformação & P7 & $\mathrm{O}$ & 1 & 1 & 6 & 18 & 26 & 1,5 & 24,5 & 26 & 0,94 & 0,06 & 0,88 & Viável \\
\hline Transformação & P8 & 2 & 1 & 4 & 4 & 15 & 26 & 5,0 & 21,0 & 26 & 0,81 & 0,19 & 0,62 & Viável \\
\hline Transformação & $\mathrm{P} 10$ & 1 & 4 & 3 & 5 & 13 & 26 & 6,5 & 19,5 & 26 & 0,75 & 0,25 & 0,50 & Dúvida \\
\hline$\sum$ Fator Transf. & & 21 & 27 & 27 & 57 & 128 & 260 & 61,5 & 198,5 & 260 & 0,76 & 0,24 & 0,53 & Dúvida \\
\hline Inovação & P11 & $\mathrm{O}$ & $\mathrm{O}$ & 0 & 7 & 19 & 26 & - & 26,0 & 26 & 1,00 & - & 1,00 & Viável \\
\hline Inovação & P12 & $\mathrm{O}$ & 1 & $\mathrm{O}$ & 5 & 20 & 26 & 1,0 & 25,0 & 26 & 0,96 & 0,04 & 0,92 & Viável \\
\hline Inovação & P13 & $\mathrm{O}$ & $\mathrm{O}$ & 2 & 3 & 21 & 26 & 1,0 & 25,0 & 26 & 0,96 & 0,04 & 0,92 & Viável \\
\hline Inovação & P14 & $\mathrm{O}$ & 2 & O & 8 & 16 & 26 & 2,0 & 24,0 & 26 & 0,92 & 0,08 & 0,85 & Viável \\
\hline Inovação & P15 & $\mathrm{O}$ & $\mathrm{O}$ & 2 & 10 & 14 & 26 & 1,0 & 25,0 & 26 & 0,96 & 0,04 & 0,92 & Viável \\
\hline Inovação & P16 & 1 & $\mathrm{O}$ & $\mathrm{O}$ & 6 & 19 & 26 & 1,0 & 25,0 & 26 & 0,96 & 0,04 & 0,92 & Viável \\
\hline Inovação & P17 & 4 & 5 & 3 & 11 & 3 & 26 & 10,5 & 15,5 & 26 & 0,60 & 0,40 & 0,19 & Dúvida \\
\hline Inovação & P18 & 1 & 2 & 3 & 13 & 7 & 26 & 4,5 & 21,5 & 26 & 0,83 & 0,17 & 0,65 & Viável \\
\hline Inovação & $\mathrm{P} 20$ & 1 & 2 & O & 5 & 18 & 26 & 3,0 & 23,0 & 26 & 0,88 & 0,12 & 0,77 & Viável \\
\hline Inovação & P21 & 1 & 2 & $\mathrm{O}$ & 6 & 17 & 26 & 3,0 & 23,0 & 26 & 0,88 & 0,12 & 0,77 & Viável \\
\hline Inovação & P22 & $\mathrm{O}$ & 2 & 2 & 3 & 19 & 26 & 3,0 & 23,0 & 26 & 0,88 & 0,12 & 0,77 & Viável \\
\hline$\sum$ Fator Inov. & & 8 & 16 & 15 & 82 & 191 & 312 & 31,5 & 280,5 & 312 & 0,90 & 0,10 & 0,80 & Viável \\
\hline Geografia da Inovaçá & $\mathrm{P} 23$ & $\mathrm{O}$ & 2 & 2 & 11 & 11 & 26 & 3,0 & 23,0 & 26 & 0,88 & 0,12 & 0,77 & Viável \\
\hline Geografia da Inovaç: & P24 & 2 & 4 & 1 & 10 & 9 & 26 & 6,5 & 19,5 & 26 & 0,75 & 0,25 & 0,50 & Dúvida \\
\hline Geografia da Inovaça & P25 & 2 & 4 & 1 & 10 & 9 & 26 & 6,5 & 19,5 & 26 & 0,75 & 0,25 & 0,50 & Dúvida \\
\hline Geografia da Inovaça & P26 & $\mathrm{O}$ & $\mathrm{O}$ & 3 & 4 & 19 & 26 & 1,5 & 24,5 & 26 & 0,94 & 0,06 & 0,88 & Viável \\
\hline Geografia da Inovaç: & $\mathrm{P} 27$ & $\mathrm{O}$ & $\mathrm{O}$ & 3 & 4 & 19 & 26 & 1,5 & 24,5 & 26 & 0,94 & 0,06 & 0,88 & Viável \\
\hline Geografia da Inovaça & P28 & 1 & 1 & 1 & 3 & 20 & 26 & 2,5 & 23,5 & 26 & 0,90 & 0,10 & 0,81 & Viável \\
\hline Geografia da Inovaça & P29 & 1 & $\mathrm{O}$ & 4 & 12 & 9 & 26 & 3,0 & 23,0 & 26 & 0,88 & 0,12 & 0,77 & Viável \\
\hline Geografia da Inovaçá & P30 & $\mathrm{O}$ & $\mathrm{O}$ & $\mathrm{O}$ & 3 & 23 & 26 & - & 26,0 & 26 & 1,00 & - & 1,00 & Viável \\
\hline Geografia da Inovaç: & P31 & $\mathrm{O}$ & $\mathrm{O}$ & $\mathrm{O}$ & 4 & 22 & 26 & - & 26,0 & 26 & 1,00 & - & 1,00 & Viável \\
\hline Geografia da Inovaçá & P32 & $\mathrm{O}$ & $\mathrm{O}$ & 2 & 8 & 16 & 26 & 1,0 & 25,0 & 26 & 0,96 & 0,04 & 0,92 & Viável \\
\hline ¿ Fator Geo. Inov. & & 6 & 11 & 17 & 69 & 157 & 260 & 25,5 & 234,5 & 260 & 0,90 & 0,10 & 0,80 & Viável \\
\hline Atores & P33 & $\mathrm{O}$ & $\mathrm{O}$ & 2 & 7 & 17 & 26 & 1,0 & 25,0 & 26 & 0,96 & 0,04 & 0,92 & Viável \\
\hline Atores & P34 & $\mathrm{O}$ & 3 & 1 & 6 & 16 & 26 & 3,5 & 22,5 & 26 & 0,87 & 0,13 & 0,73 & Viável \\
\hline Atores & P35 & $\mathrm{O}$ & 1 & 2 & 5 & 18 & 26 & 2,0 & 24,0 & 26 & 0,92 & 0,08 & 0,85 & Viável \\
\hline Atores & P36 & $\mathrm{O}$ & 1 & 1 & 11 & 13 & 26 & 1,5 & 24,5 & 26 & 0,94 & 0,06 & 0,88 & Viável \\
\hline Atores & P37 & $\mathrm{O}$ & 3 & 1 & 13 & 9 & 26 & 3,5 & 22,5 & 26 & 0,87 & 0,13 & 0,73 & Viável \\
\hline Atores & $\mathrm{P} 40$ & $\mathrm{O}$ & $\mathrm{O}$ & 1 & 10 & 15 & 26 & 0,5 & 25,5 & 26 & 0,98 & 0,02 & 0,96 & Viável \\
\hline$\sum$ Fator At. & & $\mathrm{O}$ & 10 & 9 & 67 & 122 & 208 & 14,5 & 193,5 & 208 & 0,93 & 0,07 & 0,86 & Viável \\
\hline Processo & $\mathrm{P} 41$ & $\mathrm{O}$ & 1 & 1 & 5 & 19 & 26 & 1,5 & 24,5 & 26 & 0,94 & 0,06 & 0,88 & Viável \\
\hline Processo & P42 & $\mathrm{O}$ & $\mathrm{O}$ & O & 4 & 22 & 26 & - & 26,0 & 26 & 1,00 & - & 1,00 & Viável \\
\hline Processo & $\mathrm{P} 43$ & $\mathrm{O}$ & $\mathrm{O}$ & 1 & 4 & 21 & 26 & 0,5 & 25,5 & 26 & 0,98 & 0,02 & 0,96 & Viável \\
\hline Processo & P44 & $\mathrm{O}$ & $\mathrm{O}$ & 1 & 5 & 20 & 26 & 0,5 & 25,5 & 26 & 0,98 & 0,02 & 0,96 & Viável \\
\hline Processo & P45 & $\mathrm{O}$ & $\mathrm{O}$ & 3 & 6 & 17 & 26 & 1,5 & 24,5 & 26 & 0,94 & 0,06 & 0,88 & Viável \\
\hline Processo & $P 46$ & $\mathrm{O}$ & $\mathrm{O}$ & $\mathrm{O}$ & 10 & 16 & 26 & - & 26,0 & 26 & 1,00 & - & 1,00 & Viável \\
\hline Processo & P47 & $\mathrm{O}$ & 1 & $\mathrm{O}$ & 14 & 11 & 26 & 1,0 & 25,0 & 26 & 0,96 & 0,04 & 0,92 & Viável \\
\hline Processo & P48 & $\mathrm{O}$ & 1 & $\mathrm{O}$ & 3 & 22 & 26 & 1,0 & 25,0 & 26 & 0,96 & 0,04 & 0,92 & Viável \\
\hline Processo & P49 & $\mathrm{O}$ & $\mathrm{O}$ & 1 & 5 & 20 & 26 & 0,5 & 25,5 & 26 & 0,98 & 0,02 & 0,96 & Viável \\
\hline Processo & P50 & $\mathrm{O}$ & 1 & 1 & 6 & 18 & 26 & 1,5 & 24,5 & 26 & 0,94 & 0,06 & 0,88 & Viável \\
\hline Processo & P51 & $\mathrm{O}$ & $\mathrm{O}$ & $\mathrm{O}$ & 12 & 14 & 26 & - & 26,0 & 26 & 1,00 & - & 1,00 & Viável \\
\hline Processo & P52 & 1 & 0 & 2 & 6 & 17 & 26 & 2,0 & 24,0 & 26 & 0,92 & 0,08 & 0,85 & Viável \\
\hline$\sum$ Fator Proc. & & 1 & 4 & 10 & 80 & 217 & 312 & 10 & 302 & 312 & 0,97 & 0,03 & 0,94 & Viável \\
\hline
\end{tabular}


Todos as dimensões formadas por transformação, inovação, geografia da inovação, atores e processos foram confirmadas pelos especialistas, conforme Figura 2.

Figura 2 - Quadrado Unitário do Plano Cartesiano - QUPC - Curto Prazo.

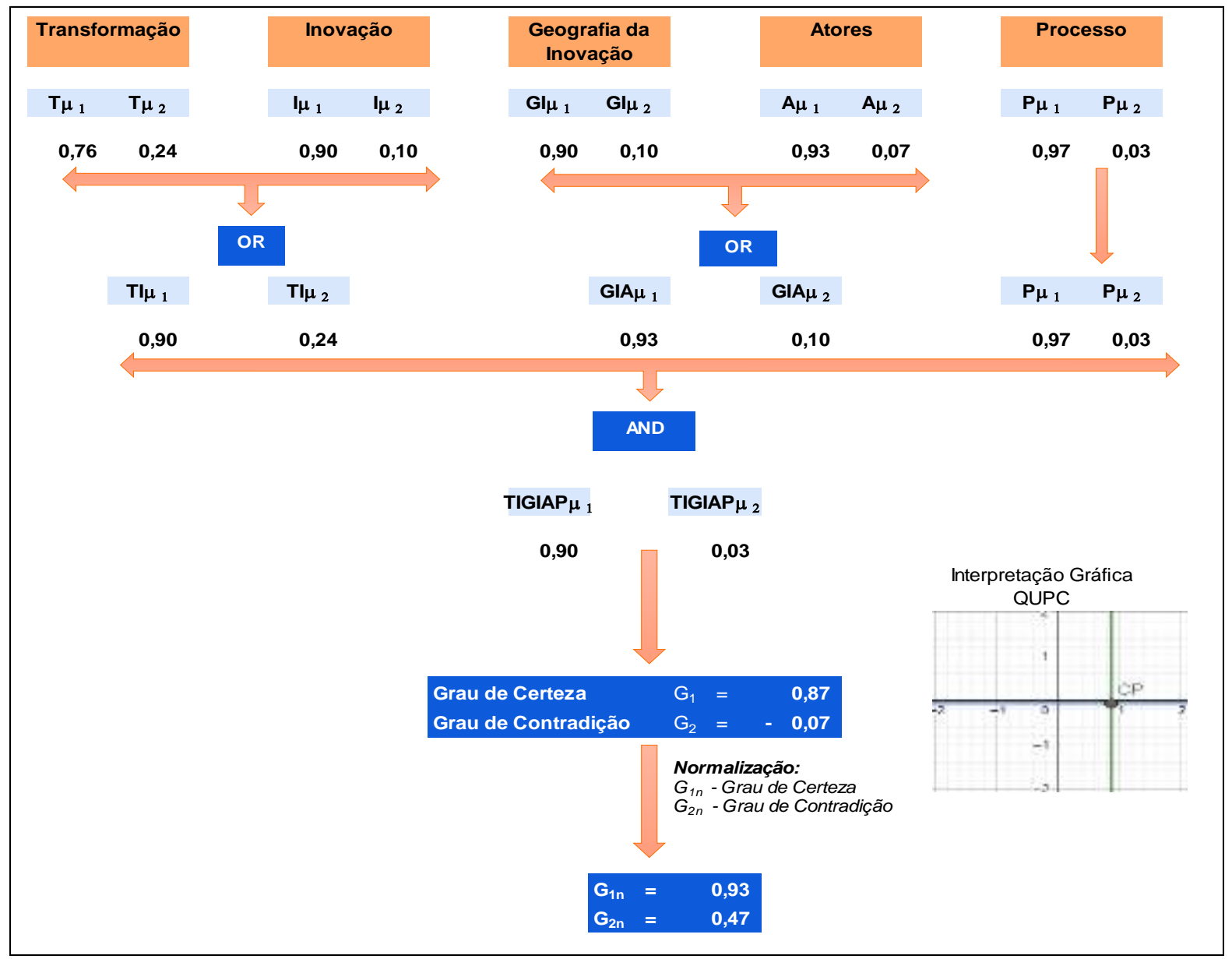

Fonte: Autores (2020).

\subsubsection{Transformação}

$\mathrm{O}$ fator transformações diz respeito às proposições relacionadas ao ambiente em que decorrem as necessidades $\mathrm{e}$ oportunidades para a inovação social (Tardif \& Harrisson, 2005). Estas proposições estão distribuídas em contexto econômico e aspectos sociais.

\subsubsection{Inovação}

A inovação está distribuída nos fatores, modelo, economia e ação social. As profundas transformações causadas principalmente pela forma como negócios são feitos, foram fortemente impactadas pelas décadas de 1990 e 2000, alguns exemplos de empresas como Uber, Netflix, Mercado Livre, Airbnb e outras, que tem como caraterística o setor de serviços, conectando prestadores de serviços diretamente a consumidores por meio de plataformas virtuais. Isto fez com que vários negócios ao redor do mundo fossem repensados, como o sistema tradicional de taxi, o segmento de hotelaria, Marketplace e locadoras de filmes. Estas disrupções provocam alterações profundas no modelo de trabalho, desenvolvimento regional e modelo de gestão. Nota-se que estes arranjos deixam de ser meramente industriais e passam a representar um poder maior de 
colaboração que envolve governo, empresas, instituições de ensino e cidadãos, quando há interação entre estes agentes, formase a vantagem colaborativa, capaz de romper com segmentos tradicionais (Kanter, 1996).

Estas inovações também ocorrem no campo social, como o Grameen Bank, o banco do povo, que atua com microcrédito para pessoas de baixa renda com o objetivo de erradicar a pobreza. Trata-se de uma empresa privada, mas que possui um modelo de negócio voltado para este público e feminino, já que $97 \%$ dos créditos são concedidos para as mulheres.

\subsubsection{Geografia da Inovação}

A geografia da inovação é orientada para a Escala, Tipos, Organização e objetivos. Os especialistas denegaram a escala nacional e internacional para o curto prazo.

No curto prazo as inovações são mais incrementais. Estas inovações incrementais podem ser definidas como mudanças que aperfeiçoam o desempenho da tecnologia, produto ou serviço, já as inovações disruptivas são formadas por novas tecnologias com efeitos sistêmicos no mercado (Hacklin, Raurich, \& Marxt, 2004).

A mudança ampla, que faz parte do conceito de inovação social (Phills, Deiglmeier, \& Miller, 2008; Tardif \& Harrisson, 2005), segundo os especialistas ocorre no médio e longo prazo.

\subsubsection{Atores}

Todos os fatores, sociais, institucionais e intermediários foram apontados como viáveis. Estes fatores estão relacionados às proposições cooperativas, organização da sociedade civil, organizações coletivas, o estado, organizações privadas, como forma de comunicação destaca-se as redes sociais alianças e parcerias, e os comitês.

O próprio conceito de inovação permite abstrair diversos agentes. Como apontado por (Drucker, 1987), nas economias desenvolvidas, a iniciativa privada foi responsável por grande parte das inovações sociais, porém ocorreram inovações no setor público como a própria constituição dos Estados Unidos que trouxe novas formas de regulamentação, diferente das anteriores e que se perpetuou até os dias atuais.

O cooperativismo, as associações, ocupam um papel especial pela sua forma de flexibilização e convívio harmonioso com vários sistemas econômicos. Surgem como alternativas de uma nova economia baseada na solidariedade, esta economia da solidariedade formada por empresas ou cooperativas surge como alternativa a pobreza por falta de oportunidade e que sobrevivem muitas vezes de transferências públicas como previdência e auxílios (Singer, 2011).

Pelos expostos, os agentes seguem determinados processos. O primeiro passo é a ideia, que pode ser construída em grupo, individual, por meio de observação do mundo ou inspirações, após isto o agente deve mobilizar outras pessoas a participarem e, por fim, capacidade de liderança para conduzir o projeto.

\subsubsection{Processos}

Os processos são formados pelos fatores modo de coordenação, meios e limitações; partilhado nas proposições efetividade, participação, mobilização, aprendizagem, parcerias, poder público e iniciativa privada. Em relação às restrições, tem-se complexidade, incerteza política, resistência às mudanças, restrição por forças internas e externas, restrição por compromisso e rigidez institucional.

Por um lado, há a liderança que deve organizar as pessoas para obter a afetividade das ações e do outro lado as limitações, que está relacionado basicamente ao quanto às pessoas estão dispostas a contribuir e quebrar paradigmas internos. Estes processos bem organizados contribuem para a busca de recursos para financiar os projetos de inovação social (Chesbrough, 2003). 


\section{Conclusão}

A Inovação Social (IS) tem seu campo fundamentado na criação de soluções frente às necessidades sociais, impulsionada por diversos agentes que se organizam sob os mais diferentes tipos jurídicos ampliando a ação das IS de um campo local para nacional ou internacional.

Um produto ou serviço de uma IS não necessariamente será gratuito, porém as regras para formação de preço não estarão amparadas no mercado tradicional, onde o preço é determinado observando a oferta e demanda, tratando-se de um mercado baseado na concorrência. O mercado social deve estar escorado na integração de diversos agentes como governo, iniciativa pública, pessoas físicas e instituições de ensino para produzir vantagem colaborativa.

Suas bases de troca de produtos e serviços estão centradas nas ideias do cooperativismo, onde a homogeneidade de interesses, acesso à tecnologia, treinamento, livre iniciativa, comprometimento, pensamento coletivo e não lucratividade, agregam diversos tipos de pessoas e empresas, independente do porte, para um objetivo comum, sendo a formação de preço concebidas para a sustentabilidade destas IS.

As IS podem ser aplicadas em qualquer modelo econômico, que provocam distúrbios sociais pela sua própria dinâmica, mesmo em economias mais desenvolvidas registra-se iniciativas de IS, denota-se um reconhecimento de diversos agentes das importâncias das IS para equilibrar estas disfunções sociais.

Isto não retira a importância do papel do Estado, mas permite que possam conviver em plena harmonia, inclusive o próprio Estado Brasileiro reconhece no caso das cooperativas e organizações sem fins lucrativos para o interesse público, ou seja, mesmo que não estejam revestidas de estado, criam produtos e serviços importantes para a sociedade, e em troca deste reconhecimento, possuem regimes diferenciados de tributação.

É considerável destacar que as IS não estão vinculadas a nenhum tipo jurídico, sendo assim cooperativas, organizações não governamentais, fundações, empresas privadas, empresas públicas e outras podem aplicar IS em suas estratégias. A maioria das inovações em países desenvolvidos foram criadas por empresas privadas.

Outro aspecto importante para a IS, diz respeito à sustentabilidade das iniciativas, com foco econômico e social. As iniciativas mais bem sucedidas tinham sua base de receita híbrida, contando com doadores, parceria pública privada, empresas e produtos sustentáveis denominados de produtos sociais. As IS que contavam apenas com doações tiveram várias iniciativas canceladas. A dependência de uma única fonte de recursos representa um risco de continuidade do projeto ou das ações, uma vez que cessam as doações por diversos motivos, estas podem ser paralisadas ou canceladas.

Do ponto de vista da liberdade, quando compreendida como um conjunto de direitos que garantam acesso amplo aos serviços públicos e garantia à vida, as IS por atuarem no interesse público, contribuem para a garantia do gozo destes direitos.

Muitas destas organizações expandiram suas operações para criar e comercializar produtos sociais, esta proposta cria uma fonte de comunicação importante com o público consumidor, além de estar em um mercado que embora se encontra em processo de reconhecimento como política macroeconômica por parte dos países e estados, já se pode pontuar que é uma onda de inovação que causa engajamento entre consumidor e organizações.

A comunicação por meio das redes sociais, a própria internet, telefonia móvel mudou a forma como estas interações ocorrem. Consumidores retratam suas experiências e conduzem a criação de valor que pode significar rupturas para as organizações ou aperfeiçoamento de suas relações, cada vez mais os produtos e serviços terão que atender a características mais específicas das pessoas, pois o consumidor está empoderado.

Este empoderamento do consumidor impõem novas dimensões aos gestores de todos os tipos de organização, conhecer estas demandas sociais e adaptá-las ao modelo de negócios são vantagens que extrapolam as fronteiras tradicionais do capital, baseadas em processos industriais e criam vantagens precípuos para ambos os stakeholders, partes interessadas. 
Sendo assim, uma IS não pode estar fundamentada no comportamento de misericórdia, pena, piedade e compaixão, pois estes comportamentos dignos de reconhecimento do ponto de vista humano prejudicam a essência da IS, que é empoderar pessoas e grupos, comunicando que todos tem potencial que pode ser estimulado para produzir produtos e serviços, este é o tipo de comportamento que promove engajamento e reconhecimento social.

Nesta perspectiva, uma IS jamais vai estigmatizar grupos sociais, por isso, como apontado pelos especialistas, a premissa de exclusão social para a composição de cenários no curto prazo foi excluída, estes são efeitos de um processo social e psicológico degenerativo, mas será resolvido ou mitigado a partir do momento em que se descubra as causas que levaram a este processo de exclusão, campo para a IS.

Portanto é significativo persistir que a inovação social embora esteja ancorada nas necessidades sociais e tem públicoalvo definido, a divulgação das iniciativas deve ocorrer para empoderar este grupo e não para criar classificações.

No cenário de curto prazo, as proposições de contexto, que se relacionam ao locus conceitual de momentos de crises e ruptura de modelo de trabalho frente à tecnologia e transformações econômicas, geraram dúvida entre os especialistas, o que demonstra que no curto prazo os aspectos são mais operacionais que estratégicos. Estes pontos em especial são relacionados a mudanças mais estruturais de médio e longo prazo. Todos os fatores, transformação, inovação, geografia da inovação, atores e processos, apresentaram grau de certeza normalizado $\left(G_{1 n}\right)$ de 0,95 e grau de contradição normalizado $\left(G_{2 n}\right)$ de 0,48 , que representa respectivamente aderência ampla e dados consistentes. Desta forma se valida o modelo para cenários prospectivos de curto prazo.

Aos pesquisadores e gestores, recomenda-se futuros novos estudos que avaliem os cenários ao longo do tempo, a fim de compreenderem como os cenários contribuem para a construção das IS e quais as vantagens e desvantagens nestes modelos, tendo como proposições: se os cenários contribuíram para aumentar o engajamento das pessoas, se os cenários fortaleceram o sistema de parcerias, se os cenários facilitaram o acesso a editais e recursos disponibilizados pela iniciativa privada e pública, e se as ações de IS geraram lealdade do consumidor.

\section{Referências}

Abe, J. M.;Costa, N. C. A. da. (1992). Fundamentos da lógica anotada (Tese (Doutorado em Filosofia)). USP.

Astigarraga, E. (2003). El Metodo Delphi. San Sebastián, Spain: Universidad de Deusto, 1-14.

Brown, J. D.. (2002). The Cronbach alpha reliability estimate. Shiken: JALT Testing \& Evaluation SIG Newsletter, 6(1), 17-18.

Budiaji, W. (2013). Skala Pengukuran Dan Jumlah Respon Skala Likert. Ilmu Pertanian Dan Perikanan, 2(2), 127-133. https://osf.io/preprints/inarxiv/k7bgy/ Carvalho, F. R.; Abe, J. M.. (2011). Tomadas de decisão com ferramentas da lógica paraconsistente anotada. Blucher.

Carvalho, F. R. de, Brustein, I., \& Abe, J. M. (2011). Tomadas de Decisão com ferramentas da Lógica Paraconsistente Anotada. XXIII Encontro Nac. de Eng. de Produção, 1, 1-8.

Chesbrough, H. W. (2003). Open innovation : the new imperative for creating and profiting from technology. Harvard Business School Publishing.

Choi, J. P., \& Thum, M. (2005). Corruption and the Shadow Economy*. International Economic Review, 46(3), 817-836. https://doi.org/10.1111/j.14682354.2005.00347.x

Costa, N. C. A. da, \& Abe, J. M. (2000). Paraconsistência em informática e inteligência artificial. Estudos Avançados, 14(39), 161-174. https://doi.org/10.1590/S0103-40142000000200012

Davis, J. A. (1976). Levantamento de Dados em Sociologia. Zahr.

Drucker, P. F. (1987). Social innovation-Management's new dimension. Long Range Planning, 20(6), 29-34. https://doi.org/10.1016/0024-6301(87)90129-4

Gastwirth, J. L., Gel, Y. R., \& Miao, W. (2009). The Impact of Levene's Test of Equality of Variances on Statistical Theory and Practice. Statistical Science, 24(3), 343-360. https://doi.org/10.1214/09-STS301

George, D., \& Mallery, P. (2018). IBM SPSS Statistics 25 Step by Step. In Taylor \& Francis. https://doi.org/10.4324/9781351033909

Hacklin, F., Raurich, V., \& Marxt, C. (2004). How incremental innovation becomes disruptive: the case of technology convergence. IEEE International 
Engineering Management Conference, 1, 32-36. https://doi.org/10.1109/IEMC.2004.1407070

Hair, J. F., Black, W. C., Babin, B. J., Anderson, R. E., \& Tatham, R. L. (2009). Analise multivariada de dados. In Bookman. Bookman.

Hamilton, D. M., \& Breslawski, S. (1996). Knowledge acquisition for multiple site, related domain expert systems: Delphi process and application. Expert Systems with Applications, 11(3), 377-389. https://doi.org/10.1016/S0957-4174(96)00052-8

Kanter, R. M. (1996). Classe Mundial, uma agenda para gerenciar os desafios globais em benefício das empresas e das comunidades. Campus.

Landis, J. R., \& Koch, G. G. (1977). The Measurement of Observer Agreement for Categorical Data. Biometrics, 33(1), 159. https://doi.org/10.2307/2529310

Levene, H. (1960). Robust testes for equality of variances. InContributions to Probability and Statistics. Stanford Univ. Press, Palo Alto, CA, I. Olkin, 278292.

Likert, R. (1932). A technique for the measurement of attitudes. In Archives of Psychology. of Psychologu.

Lind, N. (2014). Human Development Index (HDI). In Encyclopedia of Quality of Life and Well-Being Research (pp. 3012-3013). https://doi.org/10.1007/978-94-007-0753-5_1342

Macnaughton, R. J. (1996). Numbers, scales, and qualitative research. The Lancet, 347(9008), 1099-1100. https://doi.org/10.1016/S0140-6736(96)90286-X

Mannarelli Filho, T., Donadon, F. A. B., Pigatto, G. A. S., Queiróz, T. R., \& Baptista, R. D. (2021). Responsabilidade social, sustentabilidade e inovação no setor sucroenergético brasileiro: Tendências e perspectivas. Research, Society and Development, 10(4), e32610414317. https://doi.org/10.33448/rsdv10i4.14317

Mintzberg, H. (1987). The Strategy Concept I: Five Ps for Strategy. California Management Review, 30(1), 11-24. https://doi.org/10.2307/41165263

Morin, E. (2000). Os sete saberes necess á rios à educa çã o do futuro (2nd ed.); Cortez.

Papakostas, N., Papachatzakis, P., Xanthakis, V., Mourtzis, D., \& Chryssolouris, G. (2010). An approach to operational aircraft maintenance planning. Decision Support Systems, 48(4), 604-612. https://doi.org/10.1016/j.dss.2009.11.010

Phills, J. A., Deiglmeier, K., \& Miller, D. T. (2008). Rediscovering social innovation. Stanford Social Innovation Review, 6.

PNUD Brasil. (2020). Índice de Desenvolvimento Humano. https://www.br.undp.org/content/brazil/pt/home/idh0/conceitos/o-que-e-o-idh.html

Prahalad, C. K., \& Ramaswamy, V. (2004). Co-creation experiences: The next practice in value creation. Journal of Interactive Marketing, 18(3), 5-14. https://doi.org/10.1002/dir.20015

Reis, N. F. (2014). Método Paraconsistente de Cenários Prospectivos (Tese (Doutorado em Engenharia de Produção)), Universidade Paulista). https://www3.unip.br/presencial/ensino/pos_graduacao/strictosensu/eng_producao/download/eng_neliofernandodosreis.pdf

Reis, N. F., Oliveira, C. C. De, Abe, J. M., \& Andrade, A. G. (2019). Support System Decision with Logic Et applied to Merger and Acquisition - M \& A : a case study in the education industry. International Journal of Latest Engineering and Management Research (IJLEMR), 04(04), 45-50.

Rowe, G., Wright, G., \& Bolger, F. (1991). Delphi: A Re-Evaluation of Research and Theory. Technological Forecasting And Social Change, $39,235-251$.

Rozados, H. B. F. (2015). O uso da técnica Delphi como alternativa metodológica para a área da Ciência da Informação. Em Questão, 21(3), 64-86. https://seer.ufrgs.br/EmQuestao/article/download/58422/36043\%0A

Sampieri, R. H., Collado, C. F., \& Lucio, M. del P. B. (2010). Metodología de la investigación (Quinta). Colonia Desarrollo Santa Fe - McGRAW-HILL / Interamericana Editoras S.A. de C. V.

Sanches, C., Marietto, M. L., \& Paixão, M. R. (2011). Desenvolvimento e validação de questionário multidimensional, por meio de lógica paraconsistente, para medir a práxis de gestão de responsabilidade sócio ambiental. SIMPOI, $1-16 . \quad$ https://www.researchgate.net/profile/Marcio_Marietto/publication/297920574_Desenvolvimento_e_validacao_de_questionario_multidimensional_por_meio_ de_logica_paraconsistente_para_medir_a_praxis_de_festao_de_responsabilidade_socioambiental/links/56e4b14a08ae9

Sanches, C., Meireles, M., \& Sordi, J. O. de. (2011). Análise Qualitativa Por Meio da Lógica Paraconsistente: Método de Interpretação e Síntese de Informações Obtida por Escalas Likert. III Encontro de Ensino e Pesquisa Em Administração e Contabilidade.

Schwartz, P. (1996). The Art of the Long View: Planning for the Future in an Uncertain World. Doubleday Dell Publishing Group.

Singer, P. (2011). Economia solidária versus economia capitalista. Sociedade e Estado, 16(1-2), 100-112. https://doi.org/10.1590/s0102-69922001000100005

Tardif, C., \& Harrisson, D. (2005). Complémentarité, convergence et transversalité: la conceptualisation de l'innovation sociale au CRISES. Collection Études Théoriques.

Velez Pareja, I. (2003). The Delphi Method (El Metodo Delphi). SSRN Electronic Journal, 1-17. https://doi.org/10.2139/ssrn.420040

Wilkinson, R., \& Pickett, K. (2009). The Spirit Level: Why more equal societies almost always do better (Penguin, Ed.). Bloomsbury Press. 